\title{
ESTUDO COMPARATIVO DA CONSTITUIÇÃO NUTRICIONAL DA MADEIRA E CASCA DE ESPÉCIES E CLONES DE EUCALIPTO VISANDO O CULTIVO DE SHIITAKE EM TORAS ${ }^{1}$
}

\author{
Meire Cristina Nogueira de Andrade², Marli Teixeira de Almeida Minhoni ${ }^{3}$, Cláudio Angeli Sansígolo ${ }^{3}$, \\ Diego Cunha Zied ${ }^{4}$ e Ceci Sales-Campos ${ }^{5}$
}

\begin{abstract}
RESUMO - Avaliou-se as propriedades nutricionais da madeira e casca de sete espécies e três clones de eucalipto visando o cultivo de shiitake em toras. O delineamento experimental foi inteiramente casualizado, com 10 tratamentos (tipos de eucalipto) e 3 repetições, sendo cada repetição correspondente a uma amostra mista de serragem obtida de discos coletados em alturas diferentes (9cm, meio e $14 \mathrm{~cm}$ de diâmetro) de uma mesma árvore. As cascas foram removidas das cunhas de madeira para análise individual de ambas. As amostras foram encaminhadas ao Laboratório de Análise Química de Fertilizantes e Corretivos, pertencente ao Departamento de Recursos Naturais - Ciência do Solo - FCA/ UNESP, para a determinação da composição de macro e micronutrientes. O nitrogênio foi determinado pelo método de oxidação com ácido perclórico; o fósforo, através de extração nítrico-perclórico e determinação colorímetrica (método espectrofotométrico do ácido molibdovanadofosfórico); o potássio, por fotometria de chama; o carbono, por queima em mufla; o enxofre, por gravimetria e o cálcio, magnésio, sódio, cobre, ferro, manganês e zinco, por espectrofotmetria de absorção atômica. De acordo com os resultados obtidos e como esperado, verificou-se que o elemento S não foi detectado em nenhum dos tipos de eucalipto analisados, tanto para a casca como para a madeira; os elementos $\mathrm{Mg}$, $\mathrm{Na}$ e $\mathrm{Mn}$, para a madeira, apresentaram diferenças de teor entre os tipos de eucaliptos avaliados. Para a casca, $\mathrm{K}_{2} \mathrm{O}$, C, Cu e Mn demonstraram possuir diferentes teores em função do tipo de eucalipto. As cascas dos tipos de eucaliptos apresentaram teores médios elevadíssimos de Ca (77x), K (3,7x), Mg (39x), Na (2,5x), Mn (26x) e Fe (1,9x) em relação às correspondentes madeiras (o elemento predominante na casca foi o $\mathrm{Ca}$ ). Os elementos predominantes na madeira foram $\mathrm{Ca}, \mathrm{K}, \mathrm{Mg}$ e $\mathrm{P}$.
\end{abstract}

Palavras-chave: Eucalipto, Nutrientes, Lentinula edodes.

\section{COMPARATIVE STUDY OF THE NUTRITIONAL CONSTITUTION OF WOOD AND BARK OF SPECIES AND CLONES OF EUCALYPTUS AIMING THE CULTIVATION OF SHIITAKE IN LOGS}

\begin{abstract}
The nutritional properties of wood and bark of seven species and three clones of eucalyptus were evaluated, aiming the cultivation of shiitake in logs. The experimental design was totally randomized, with 10 treatments (types of eucalyptus) and 3 repetitions, being each repetition corresponding to a mixed sample of sawdust obtained from discs collected in different heights $(9 \mathrm{~cm}$, middle and $14 \mathrm{~cm}$ of diameter) from the same tree. Barks were removed from wood wedges for individual analysis. Samples were sent to the Laboratory of Chemical Analysis of Fertilizers and Correctives of the Department of Natural Resources - Soil Science - School of Agronomic Sciences/UNESP, for determination of macro and micronutrients composition. Nitrogen determination was performed by oxidation with perchloric acid; phosphorus was
\end{abstract}

\footnotetext{
${ }^{1}$ Recebido em 06.05.2008 e aceito para publicação em 16.12.2010.

${ }^{2}$ Bolsista de Pós-Doutorado, CAPES, Programa de Pós Graduação em Energia na Agricultura, Faculdade de Ciências Agronômicas, UNESP, Botucatu, SP, Brasil. E-mail: <mcnandrade@hotmail.com>.

${ }^{3}$ Docentes da Faculdade de Ciências Agronômicas, UNESP, Botucatu, SP, Brasil. E-mail: <marliminhoni@fca.unesp.br> e <sansigolo@fca.unesp.br>.

${ }^{4}$ Doutorando em Agronomia, Faculdade de Ciências Agronômicas, UNESP, Botucatu, SP, Brasil. E-mail: <dczied@fca.unesp.br>. ${ }^{5}$ Pesquisadora do Instituto Nacional de Pesquisas da Amazônia, INPA, Manaus, AM, Brasil. E-mail: <ceci@inpa.gov.br> .
} 
determined with nitric-perchloric extraction and colorimetric determination (spectrophotometric molybdovanadophosphoric acid method); potassium was determined by flame photometry; carbon, by muffle burning; sulphur, by gravimetry and calcium, magnesium, sodium, copper, iron, manganese and zinc, by atomic absorption spectrophotometry. According to the results obtained and as expected, it was verified that the element $S$ was not detected in any of the kinds of eucalyptus analyzed, both for bark and wood; for wood, elements $\mathrm{Mg}, \mathrm{Na}$ and Mn presented difference in contents among eucalyptus types analyzed. For bark, $\mathrm{K}_{2} \mathrm{O}, \mathrm{C}, \mathrm{Cu}$ and $\mathrm{Mn}$ showed different contents in function of eucalyptus type. Barks of eucalyptus types presented very high contents of Ca (77x), K (3,7x), Mg (39x), Na (2,5x), Mn (26x) e Fe (1,9x), compared to the corresponding woods (Ca was the prevailing element in bark). Ca, K, Mg and P were the prevailing elements in wood.

Keywords: Eucalyptus, Nutrients and Lentinula Edodes.

\section{INTRODUÇÃO}

O cultivo de shiitake (Lentinula edodes) em toras é o método tradicional ainda muito utilizado pelos fungicultores devido à simplicidade e baixo investimento inicial (ANDRADE; GRACIOLLI, 2005; ANDRADE et al., 2002). A escolha da espécie arbórea deve ser feita em função de suas características químicas e físicas e de fatores econômicos e ambientais (ANDRADE et al., 2007; SAN ANTONIO, 1981). Entre as espécies do Brasil, o eucalipto tem sido o mais utilizado em razão, principalmente, da sua maior disponibilidade e seu efeito ecológico na preservação das espécies florestais nativas.

As espécies de eucalipto mais utilizadas no Brasil têm sido o Eucalyptus grandis (55\%), o Eucalyptus saligna Sm. (17\%), o Eucalyptus urophylla S.T. Blake (9\%) e outras espécies (8\%), bem como os híbridos de Eucalyptus grandis x E. urophylla (11\%) (SILVA, 2003). O gênero Eucalyptus possui variabilidade genética elevada. São centenas de espécies com propriedades físicas e químicas tão diversas que fazem com que os eucaliptos sejam utilizados nas mais diversas finalidades (SILVA, 2003). Apesar de a maior parte das florestas estar comprometida com a produção de madeira para os denominados “usos tradicionais” (celulose, papel, carvão vegetal, lenha e chapas de fibras), o eucalipto também vem sendo utilizado com sucesso para a produção do L. edodes (ANDRADE et al., 2008; ANDRADE, 2007; QUEIROZ, 2002; TEIXEIRA, 2000; MONTINI, 1997).

As características químicas da madeira variam com os tecidos, de modo que a distribuição dos compostos químicos é heterogênea, como resultado da estrutura anatômica da planta. Cada componente está presente em quantidades específicas (TREVISAN et al., 2007; SOUZA et al., 1979) e possui características bem definidas, as quais podem ser influenciadas pelas condições a que a madeira está submetida (BROWNING, 1963). Assim, para o cultivo do L. edodes, recomenda-se que as árvores sejam obtidas preferencialmente de áreas adubadas e que sejam de primeiro ou de segundo corte (MINHONI et al., 2007; TOKIMOTO, 2005; PRZYBYLOWICZ e DONOGHUE, 1990), pois nessas condições há maior concentração de nutrientes, como N, P, K, Ca, Mo, entre outros (BARROS, 1990), favorecendo o desenvolvimento do L. edodes. Também há grande variabilidade na produção do $L$. edodes em função das espécies de eucalipto, mesmo que provenientes de povoamentos que tiveram adubação em seu manejo, sendo tais resultados dependentes das características químicas e físicas de cada espécie utilizada (TEIXEIRA, 2000).

O conteúdo dos componentes minerais da madeira é normalmente baixo, formado, principalmente, por óxidos como: óxido de cálcio, de magnésio, de fósforo, de silício, de potássio e outros (BROWNING, 1963). O teor de cinzas varia entre 0,2 e $1 \%$ do peso de matéria seca das madeiras de clima temperado, podendo atingir $5 \%$ para as de clima tropical (FENGEL; WEGENER, 1984). Especificamente para a madeira do gênero Eucalyptus, o teor de cinzas raramente chega a 1\% do seu peso seco (TSOUMIS, 1991).

Há certa dependência do teor de minerais e da composição em função das condições ambientais (solo e clima) e da localização dentro da árvore (FENGEL; WEGENER, 1984). O teor de elementos minerais é decrescente na seguinte ordem: folha, casca, raiz, ramo e tronco.

Fengel e Wegener (1984) relataram que os principais componentes das cinzas da madeira são cálcio, potássio e magnésio. Em muitas madeiras, o Ca representa 50\% 
de todos os elementos presentes nas cinzas da madeira. O K e o Mg estão em segundo e terceiro lugares, respectivamente, seguidos por $\mathrm{Mn}, \mathrm{Na}, \mathrm{P} \mathrm{e} \mathrm{Cl}$. A madeira contém muitos outros elementos com concentração inferior a 50 ppm. Os 12 elementos traços são: Ba, Al, Fe, Zn, Cu, Ti, Pb, Ni, V, Co, Ag e Mo. Usualmente, a casca tem muito mais componentes minerais que a madeira correspondente. A frequência dos elementos é também diferente na madeira. Geralmente, o teor de cinzas na casca de diversas madeiras é superior a 10\%, ou seja, cerca de 10 vezes mais elevado que na madeira. O elemento predominante é o cálcio (82-95\%); o potássio e o magnésio ocorrem em quantidades secundárias. Em muitos casos, a quantidade dos outros elementos é inferior a $1 \%$ da soma de todos os outros.

Allison et al. (2000) estudaram o perfil de metais em madeiras de coníferas e folhosas, provenientes da operação de polpação Kraft no Sudeste dos Estados Unidos. As análises por hidrólise ácida em amostras de cavacos de madeira sugeriram que a concentração relativa de metais foi: $\mathrm{Ca}>\mathrm{K}>\mathrm{Mg}>\mathrm{Mn}>(\mathrm{Na}, \mathrm{Ba})$ $>$ Fe. Diferenças foram notadas entre amostras de cavacos de coníferas e folhosas. As análises de amostras da casca indicaram que o material tem significativamente valores mais elevados de $\mathrm{Ca}, \mathrm{K}$ e $\mathrm{Mg}$ que os correspondentes cavacos de madeira.

O eucalipto caracteriza-se por relação C/N/P elevada, próxima a 200/1/0,2. Essa relação elevada pode explicar o porquê de a produtividade do cogumelo ser maior nos primeiros fluxos de produção, diminuindo ao longo dos demais fluxos. À medida que os fluxos ocorrem, tem-se diminuição na concentração de nutrientes, como o nitrogênio e o fósforo, em decorrência da absorção desses pelo micélio do L. edodes (TEIXEIRA, 2000).

Alguns elementos químicos como o C, N, P, K e Mg são considerados “essenciais” para os fungos e absorvidos em maior quantidade, enquanto outros, por necessitarem de pequenas quantidades, são considerados “traços” (Fe, Zn, Mn, Cu e Mo), e alguns elementos químicos são detectados na constituição dos fungos, porém não indicam necessariamente algum papel biológico (MILES; CHANG, 1997).

De acordo com Eira e Montini (1997), a casca armazena cerca de $50 \%$ dos nutrientes das toras e esse fato favorece o desenvolvimento do fungo. O cerne, por sua vez, por conter concentração menor de água e de nutrientes, além de teores elevados de compostos inibidores do crescimento do fungo, não deve exceder $30 \%$ do diâmetro da tora.

Assim, objetivou-se com este trabalho fazer um estudo comparativo da constituição nutricional da madeira e casca de sete espécies e três clones de eucalipto, visando o cultivo de shiitake em toras.

\section{MATERIAL E MÉTODOS}

O experimento foi conduzido nas dependências do Departamento de Produção Vegetal, da Faculdade de Ciências Agronômicas (FCA), da Universidade Estadual Paulista (UNESP), localizada na Fazenda Experimental Lageado, Campus de Botucatu, SP.

As espécies de eucalipto utilizadas nos experimentos foram: E. saligna, E. grandis, E. urophylla, E. camaldulensis, E. citriodora, E. paniculata e E. pellita. Os clones, por sua vez, foram híbridos de E. grandis x E. urophylla, desenvolvidos por duas empresas de celulose e papel e identificados como clones 23, 24 e 25. Tanto as espécies quanto os clones de eucalipto foram obtidos na Estação Experimental de Ciências Florestais, ESALQ/ USP, Itatinga, SP. As árvores utilizadas tinham 8 anos de idade e foram retiradas de uma mesma área de plantio, cultivadas em Latossolo Vermelho-Amarelo, no espaçamento de 3,0 x 1,8 m.

O delineamento experimental foi inteiramente casualizado com 10 tratamentos (tipos de eucalipto) e três repetições (amostra mista de serragem obtida de discos coletados em alturas diferentes de uma mesma árvore).

Inicialmente, para cada espécie e clone de eucalipto foram selecionadas ao acaso três árvores, totalizando 30 [( 7 espécies +3 clones de eucalipto) x 3 árvores]. Essas árvores foram derrubadas e foram retirados de cada uma três discos em alturas distintas com 2,5 cm de espessura. Para tanto, com o auxílio de uma suta foram delimitados dois extremos em cada árvore, um com 9 e outro com $14 \mathrm{~cm}$ de diâmetro, de onde foi removido um disco por extremidade. O terceiro disco foi retirado do meio dessa área delimitada. Assim, o total de discos coletados foi 90 [(7 espécies + 3 clones de eucalipto) x 3 árvores x 3 discos], os quais foram identificados ainda no campo. Posteriormente, cada disco foi dividido em quatro cunhas com ângulo de $90^{\circ}$ entre si. A primeira cunha foi utilizada para a determinação de nutrientes e as demais cunhas, guardadas como reserva.

Revista Árvore, Viçosa-MG, v.35, n.2, p.183-192, 2011 
Posteriormente, foram removidas as cascas das cunhas para análise individual de ambas. Na sequência, três cunhas de cada árvore (coletadas de alturas distintas da árvore: $9 \mathrm{~cm}$, meioe $14 \mathrm{~cm}$ ) foram agrupadas ereduzidas manualmente a cavacos e, depois, a palitos, constituindo 30 amostras de madeira e 30 amostras de casca, as quais foram utilizadas na determinação do teor de nutrientes. Finalmente, as amostras foram reduzidas a serragem em macromoinho Willey. A serragem obtida foi classificada em peneiras para obtenção da fração 40/60 mesh. Portanto, as amostras de madeira e casca utilizadas nas análises foram compostas de cunhas obtidas em alturas distintas das árvores.

As amostras foram encaminhadas ao Laboratório de Análise Química de Fertilizantes e Corretivos, pertencente ao Departamento de Recursos Naturais - Ciência do Solo - FCA/ UNESP para determinação da composição de macro e micronutrientes, de acordo com a metodologia do Lanarv (1988). O nitrogênio foi determinado pelo método de oxidação com ácido perclórico; o fósforo, através de extração nítrico-perclórico e determinação colorímetrica (método espectrofotométrico do ácido molibdovanadofosfórico); o potássio, por fotometria de chama; o carbono, por queima em mufla; o enxofre, por gravimetria e o cálcio, magnésio, sódio, cobre, ferro, manganês e zinco, por espectrofotometria de absorção atômica.

Os dados foram submetidos à análise de variância e as médias, comparadas pelo teste de Tukey (5\%) (SNEDECOR e COCHRAN, 1972). Para tanto, utilizou-se o programa SISVAR 4.2, desenvolvido pelo Departamento de Ciências Exatas da Universidade Federal de Lavras, MG.

\section{RESULTADOS}

A Tabela 1 ilustra o teor de nutrientes na madeira e casca dos diferentes tipos de eucalipto, logo após o corte.

Tabela 1 - Resultados da análise de nutrientes da madeira e da casca do E. saligna (SAL), E. grandis (GRA), clone 23* (C23), clone 25 (C25), clone 24 (C24), E. urophylla (URO), E. pellita (PEL), E. paniculata (PAN), E. citriodora (CIT) e de E. camaldulensis (CAM), logo após o corte.

Table 1 - Results of the analysis of nutrients of wood and bark of the E. saligna (SAL), E. grandis (GRA), clone 23*(C23), clone 25 (C25), clone 24 (C24), E. urophylla (URO), E. pellita (PEL), E. paniculata (PAN), E. citriodora (CIT) and of E. camaldulensis (CAM), soon after the cut.

\begin{tabular}{|c|c|c|c|c|c|c|c|c|c|c|c|c|}
\hline \multirow[t]{2}{*}{ Eucaliptos } & & \multicolumn{11}{|c|}{ Madeira } \\
\hline & $\mathrm{C}$ & $\mathrm{N}$ & $\mathrm{P}_{2} \mathrm{O}_{5}$ & $\mathrm{~K}_{2} \mathrm{O}$ & $\mathrm{Ca}$ & $\mathrm{Mg}$ & $\mathrm{S}$ & $\mathrm{Na}$ & $\mathrm{Cu}$ & $\mathrm{Fe}$ & $\mathrm{Mn}$ & $\mathrm{Zn}$ \\
\hline & $\%$ & \multicolumn{11}{|c|}{$\mathrm{mg} / \mathrm{kg}$} \\
\hline SAL & 53,9 & 1850 & 250 & 600 & 300 & 150 & 0 & 153 & 0 & 56 & 7 & 12 \\
\hline GRA & 53,7 & 1800 & 217 & 567 & 217 & 133 & 0 & 150 & 0 & 49 & 4 & 3 \\
\hline C23 & 53,6 & 1533 & 183 & 550 & 183 & 150 & 0 & 197 & 19 & 39 & 7 & 94 \\
\hline C25 & 53,1 & 1733 & 233 & 567 & 250 & 200 & 0 & 137 & 0 & 36 & 4 & 2 \\
\hline $\mathrm{C} 24$ & 53,7 & 1700 & 350 & 750 & 233 & 150 & 0 & 190 & 10 & 53 & 13 & 63 \\
\hline URO & 53,7 & 1550 & 267 & 367 & 133 & 83 & 0 & 240 & 19 & 34 & 4 & 88 \\
\hline PEL & 53,4 & 1767 & 283 & 617 & 650 & 167 & 0 & 223 & 13 & 20 & 9 & 57 \\
\hline PAN & 53,9 & 1700 & 350 & 667 & 433 & 167 & 0 & 200 & 20 & 25 & 30 & 83 \\
\hline CIT & 53,2 & 2050 & 350 & 650 & 567 & 233 & 0 & 393 & 12 & 31 & 21 & 57 \\
\hline CAM & 53,3 & 1833 & 350 & 783 & 283 & 133 & 0 & 216 & 4 & 57 & 25 & 5 \\
\hline \multirow[t]{2}{*}{ Média } & 53,5 & 1752 & 283 & 612 & 325 & 157 & 0 & 210 & 10 & 40 & 12 & 46 \\
\hline & & \multicolumn{11}{|c|}{ Casca } \\
\hline SAL & 49,5 & 2583 & 750 & 2733 & 29033 & 7633 & 0 & 497 & 6 & 73 & 227 & 64 \\
\hline GRA & 50,7 & 2250 & 667 & 2800 & 27267 & 6767 & 0 & 420 & 0 & 65 & 147 & 44 \\
\hline C23 & 49,2 & 2450 & 167 & 2700 & 28633 & 6567 & 0 & 653 & 4 & 65 & 861 & 44 \\
\hline C25 & 50,1 & 2683 & 317 & 2200 & 29017 & 7167 & 0 & 587 & 0 & 75 & 247 & 46 \\
\hline C24 & 50,4 & 2367 & 183 & 2000 & 34433 & 9267 & 0 & 683 & 1 & 103 & 208 & 36 \\
\hline URO & 50,8 & 2600 & 283 & 2317 & 19900 & 5100 & 0 & 643 & 16 & 104 & 250 & 47 \\
\hline PEL & 50,7 & 3017 & 233 & 1800 & 13917 & 3900 & 0 & 610 & 11 & 105 & 182 & 27 \\
\hline PAN & 49,4 & 2650 & 100 & 2000 & 19667 & 4233 & 0 & 437 & 25 & 83 & 266 & 43 \\
\hline CIT & 49,1 & 2150 & 117 & 1900 & 18667 & 3467 & 0 & 237 & 13 & 26 & 351 & 77 \\
\hline CAM & 48,6 & 2317 & 383 & 2400 & 30217 & 6750 & 0 & 460 & 19 & 61 & 551 & 51 \\
\hline Média & 48,0 & 2507 & 320 & 2285 & 25075 & 6085 & 0 & 523 & 10 & 76 & 329 & 48 \\
\hline
\end{tabular}

*Os clones 23, 24 e 25 são híbridos de E. grandis x E. urophylla.

Revista Árvore, Viçosa-MG, v.35, n.2, p.183-192, 2011 
Na Tabela 2, encontram-se os valores de F obtidos na análise de variância dos dados de macro e micronutrientes da madeira (sem casca) de 10 tipos de eucalipto, logo após o corte. Houve efeito significativo do tipo de eucalipto apenas nas variáveis teor de magnésio (Mg), sódio (Na) e manganês (Mn).
Na Tabela 3, encontram-se os valores de F obtidos na análise de variância dos dados de macro e micronutrientes da casca de 10 tipos de eucalipto, logo após o corte. Houve efeito significativo do tipo de eucalipto nas variáveis monóxido de potássio $\left(\mathrm{K}_{2} \mathrm{O}\right)$, carbono (C), cobre $(\mathrm{Cu})$ e manganês $(\mathrm{Mn})$.

Tabela 2 - Valores de F obtidos na análise de variância de macro e micronutrientes da madeira (sem casca) de sete espécies (E. saligna, E. grandis, E. urophylla, E. pellita, E. paniculata, E. citriodora e E. camaldulensis) e três clones (híbridos de E. urophylla x E. grandis) de eucalipto logo após corte.

Table 2 -Values of F obtained in the analysis of variance of macronutrients and micronutrients of the wood (without bark) of seven species (E. saligna, E. grandis, E. urophylla, E. pellita, E. paniculata, E. citriodora e E. camaldulensis) and three clones (hybrid of $\mathbf{E}$. urophylla $x$ E. grandis) of eucalyptus soon after cut.

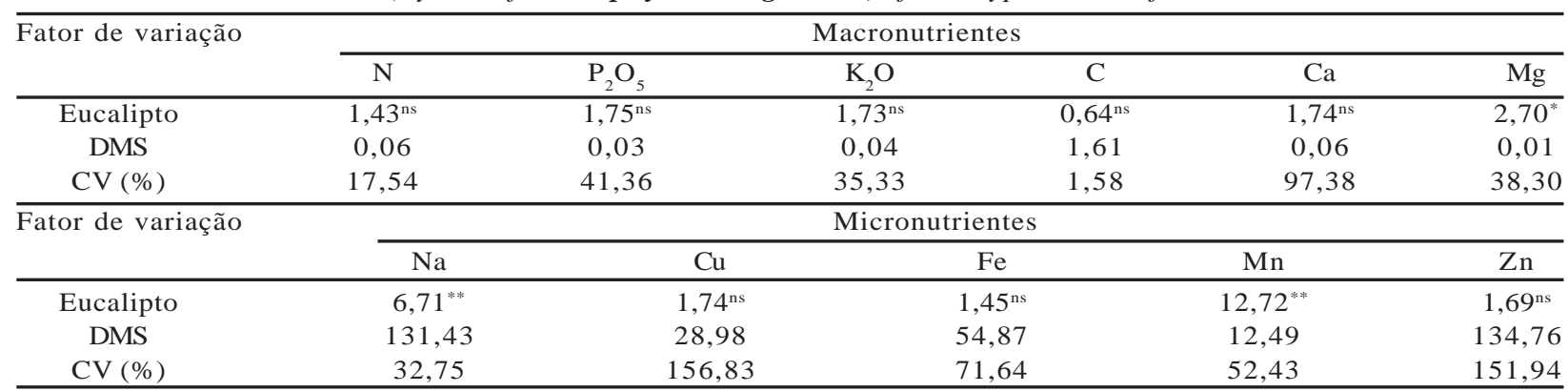

*Significativo ao nível de 5\%; ${ }^{* *}$ Significativo ao nível de 1\%; ns: não significativo.

Tabela 3 - Valores de F obtidos na análise de variância de macro e micronutrientes da casca de sete espécies (E. saligna, E. grandis, E. urophylla, E. pellita, E. paniculata, E. citriodora e E. camaldulensis) e três clones (híbridos de E. urophylla x E. grandis) de eucalipto logo após corte.

Table 3 - Values of F obtained in the analysis of variance of macronutrients and micronutrients of the bark of seven species (E. saligna, E. grandis, E. urophylla, E. pellita, E. paniculata, E. citriodora e E. camaldulensis) and three clones (hybrid of E. urophylla $\times$ E. grandis) of eucalyptus soon after cut.

\begin{tabular}{|c|c|c|c|c|c|c|}
\hline \multirow[t]{2}{*}{ Fator de variação } & \multicolumn{6}{|c|}{ Macronutrientes } \\
\hline & $\mathrm{N}$ & $\mathrm{P}_{2} \mathrm{O}_{5}$ & $\mathrm{~K}_{2} \mathrm{O}$ & $\mathrm{C}$ & $\mathrm{Ca}$ & $\mathrm{Mg}$ \\
\hline Eucalipto & $1,97^{\mathrm{ns}}$ & $1,95^{\mathrm{ns}}$ & $3,44 * *$ & $3,01 * *$ & $0,97^{\mathrm{ns}}$ & $0,66^{\mathrm{ns}}$ \\
\hline DMS & 0,07 & 0,05 & 0,09 & 2,15 & 1,89 & 0,54 \\
\hline CV (\%) & 15,15 & 84,12 & 21,42 & 2,25 & 39,49 & 46,66 \\
\hline \multirow[t]{2}{*}{ Fator de variação } & \multicolumn{6}{|c|}{ Micronutrientes } \\
\hline & $\mathrm{Na}$ & $\mathrm{Cu}$ & & $\mathrm{Fe}$ & Mn & $\mathrm{Zn}$ \\
\hline Eucalipto & $0,94^{\mathrm{ns}}$ & $7,01 * *$ & & $0,57^{\mathrm{ns}}$ & $3,81 * *$ & $1,66^{\mathrm{ns}}$ \\
\hline DMS & 380,65 & 15,58 & & 81,71 & 524,27 & 50,60 \\
\hline CV (\%) & 38,10 & 85,20 & & 56,39 & 83,37 & 55,17 \\
\hline
\end{tabular}

**Significativo ao nível de 1\%; ns: não significativo.

Na Figura 1 (A) é ilustrada a comparação da média de magnésio da madeira (sem casca) em função do tipo de eucalipto, logo após o corte. As maiores médias foram observadas no clone 25 e no E. citriodora. Médias intermediárias foram verificadas em E. saligna, E. grandis, nos clones 23 e 24, em E. pellita, E. paniculata e E. camaldulensis. Entretanto, a menor média ocorreu no E. urophylla.
Na Figura 1 (B e C) são ilustradas as comparações das médias do teor de sódio e de manganês da madeira (sem casca) em função do tipo de eucalipto, logo após o corte. Devido ao fato de as médias dos outros micronutrientes da madeira (sem casca), logo após o corte, não terem diferido significativamente entre si, elas não foram discutidas neste trabalho; apenas citadas (Tabela 1). 
*Os clones 23, 24 e 25 são híbridos de E. grandis x E. urophylla. *The clones 23, 24 and 25 are hybrid of $\boldsymbol{E}$. grandis $x \boldsymbol{E}$. urophylla.
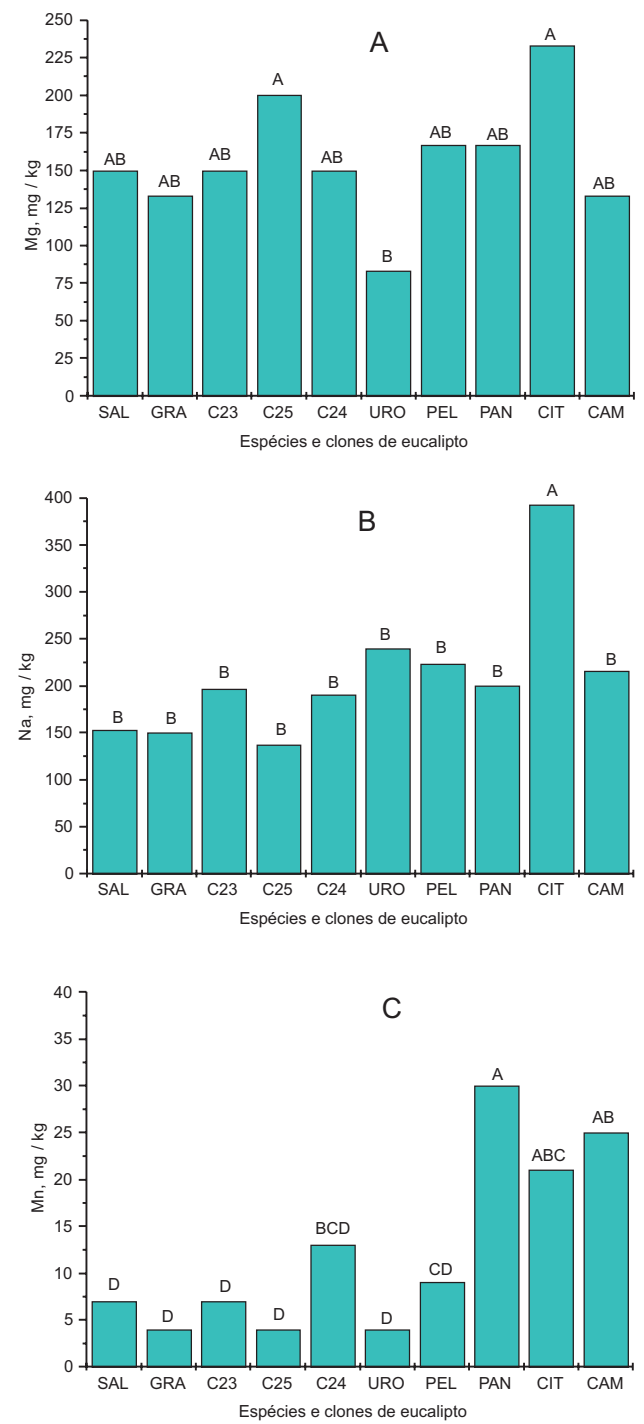

Figura 1 - Teor de: A. magnésio; B. sódio; e C. manganês da madeira de E. saligna (SAL), E. grandis (GRA), clone 23* (C23), clone 25 (C25), clone 24 (C24), E. urophylla (URO), E. pellita (PEL), E. paniculata (PAN), E. citriodora (CIT) e de E. camaldulensis (CAM) logo após o corte. Médias com letras iguais não diferem entre si (Tukey, 5\%).

Figure 1 - Content of: A. magnesium; B. sodium; and C. manganese of the wood of E. saligna ( $S A L)$, E. grandis (GRA), clone 23* (C23), clone 25 (C25), clone 24 (C24), E. urophylla (URO), E. pellita (PEL), E. paniculata $(P A N)$, E. citriodora (CIT) and of E. camaldulensis (CAM) soon after the cut. Averages with same letters don't differ amongst themselves (Tukey, 5\%).
A Figura 1 (B) mostra a comparação de médias do teor de sódio da madeira (sem casca) de 10 tipos de eucalipto, logo após o corte. Verificou-se que a maior média foi observada no E. citriodora, a qual diferiu significativamente de todos os demais tipos de madeiras analisadas.

A figura 1 (C) mostra a comparação de médias do teor de manganês da madeira (sem casca) de 10 tipos de eucalipto, logo após o corte. As maiores médias foram observadas no E. paniculata, E. citriodora e $E$. camaldulensis. No entanto, as menores médias foram observadas no E. saligna, E. grandis, clone 23, clone 25 e E. urophylla, clone 24 e E. pellita.

Na Figura 2 (A e B) são ilustradas as comparações das médias do teor de monóxido de potássio e carbono da casca em função do tipo de eucalipto, após oito meses de incubação com o L. edodes. Devido ao fato de as médias dos outros macronutrientes da casca, logo após o corte, não terem diferido significativamente entre si, elas não foram discutidas neste trabalho; apenas citadas (Tabela 1).

A Figura 2 (A) mostra a comparação de médias do teor de monóxido de potássio da casca de 10 tipos de eucalipto, logo após o corte. A maior média foi observada no E. grandis. Médias intermediárias foram obtidas pelo E. saligna, clone 23, clone 25, clone 24, E. urophylla, E. paniculata e E. camaldulensis. Entretanto, as menores médias foram observadas no E. pellita e no E. citriodora.

A Figura 2 (B) mostra a comparação de médias do teor de carbono da casca de 10 tipos de eucalipto, logo após o corte. A maior média foi verificada no E. urophylla. Médias intermediárias foram obtidas no E. saligna, E. grandis, clone 23, clone 25, clone 24, E. pellita, E. paniculata e no E. citriodora. Finalmente, a menor média foi encontrada no $E$. camaldulensis.

Na Figura 2 (C e D) são ilustradas as comparações das médias do teor de cobre e de manganês da casca em função do tipo de eucalipto, logo após o corte. Em razão das médias dos outros micronutrientes da casca, logo após o corte, não terem diferido significativamente entre si, elas não foram discutidas neste trabalho, apenas citadas (Tabela 1). A Figura 2 (C) ilustra a comparação de médias do teor de cobre da casca de 10 tipos de eucalipto, logo após o corte. 
*Os clones 23, 24 e 25 são híbridos de E. grandis x E. urophylla.

* The clones 23, 24 and 25 are hybrid of E. grandis $x$ E. urophylla.
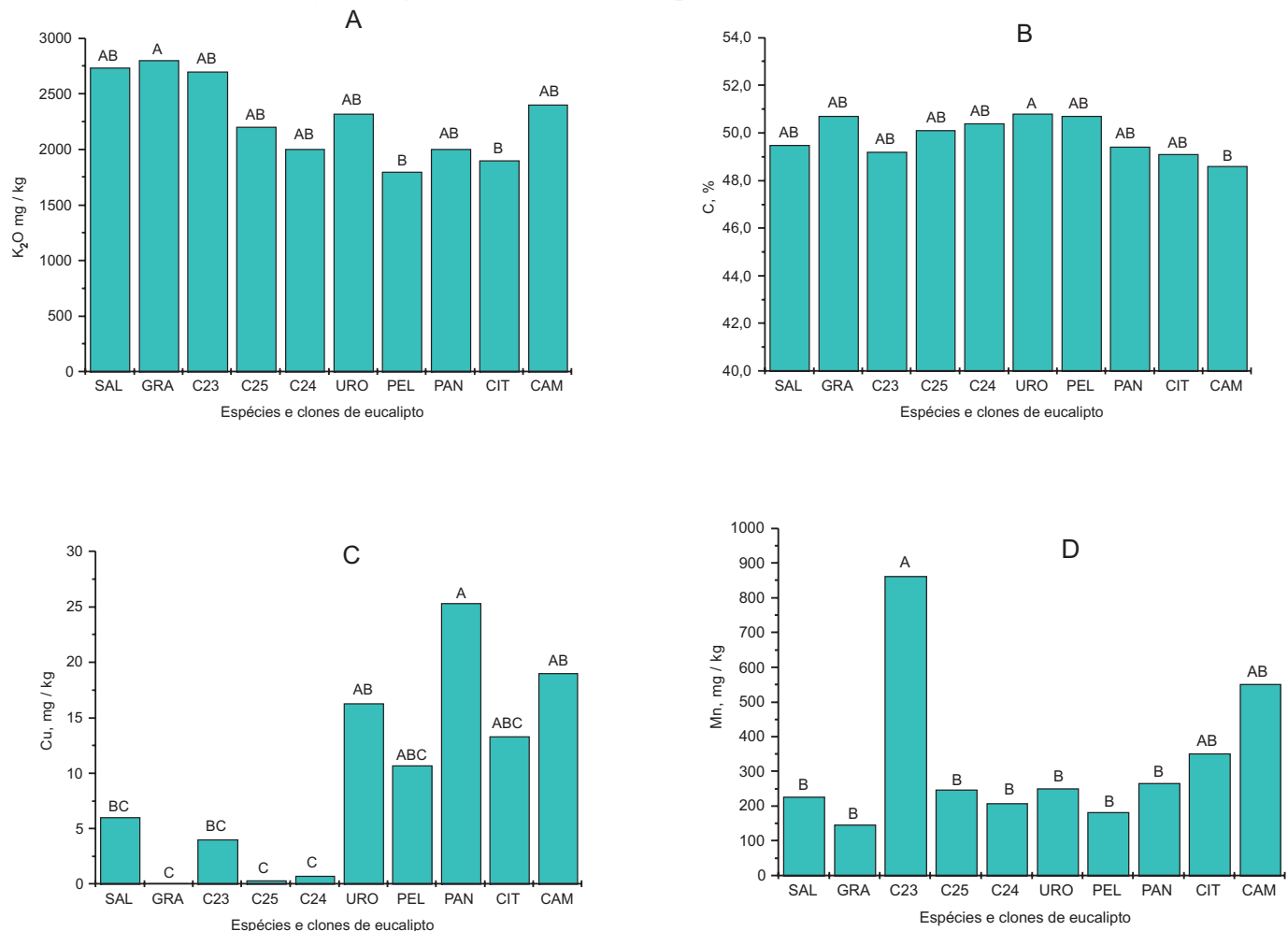

Figura 2 - Teor de: A. monóxido de potássio; B. carbono; C. cobre; e D. manganês da casca de E. saligna (SAL), E. grandis (GRA), clone 23* (C23), clone 25 (C25), clone 24 (C24), E. urophylla (URO), E. pellita (PEL), E. paniculata (PAN), E. citriodora (CIT) e de E. camaldulensis (CAM) logo após o corte. Médias com letras iguais não diferem entre si (Tukey, 5\%).

Figure 2- Content of: A. Potassium monoxide; B. carbon; C. copper; and D. manganese of the bark of E. saligna (SAL), E. grandis (GRA), clone 23* (C23), clone 25 (C25), clone 24 (C24), E. urophylla (URO), E. pellita (PEL), E. paniculata (PAN), E. citriodora (CIT) and of E. camaldulensis (CAM) soon after the cut. Averages with same letters don't differ amongst themselves (Tukey, 5\%).

As maiores médias foram verificadas no $E$. paniculata, E. urophylla e no E. camaldulensis. Médias intermediárias foram observadas no E. pellita e no $E$. citriodora. No entanto, as menores médias foram observadas no E. grandis, clone 25, clone 24, E. saligna e clone 23.

A Figura 2 (D) ilustra a comparação de médias do teor de manganês da casca de 10 tipos de eucalipto, logo após o corte. A maior média foi observada no clone 23 e médias intermediárias, no E. citriodora e no E. camaldulensis. Finalmente, as menores médias ocorreram no E. saligna, E. grandis, clone 25, clone 24, E. urophylla, E. pellita e E. paniculata.

\section{DISCUSSÃO}

Os macronutrientes presentes na madeira (sem a casca) logo após o corte (Tabela 1) obedeceram, de forma geral, à seguinte ordem decrescente: $\mathrm{C}>\mathrm{N}>$ $\mathrm{K}_{2} \mathrm{O}>\mathrm{Ca}>\mathrm{P}_{2} \mathrm{O}_{5}>\mathrm{Mg}$, enquanto os micros obtiveram a seguinte: $\mathrm{Na}>\mathrm{Zn}>\mathrm{Fe}>\mathrm{Mn}>\mathrm{Cu}$. A presença desses minerais em todas as amostras de madeira, de acordo com Molena (1986), Chang e Miles (1989) e Miles e Chang (1997), é importante para o cultivo de cogumelos.

Entre os nutrientes, o nitrogênio tem atraído particular atenção. Acredita-se que a correlação entre a concentração na madeira e a suscetibilidade da madeira

Revista Árvore, Viçosa-MG, v.35, n.2, p.183-192, 2011 
à decomposição possa ser influenciada pela taxa de nitrogênio, sendo então um dos fatores limitantes no desenvolvimento do crescimento do fungo (BOYLE, 1998). No entanto, o teor de $\mathrm{N}$ dos tipos de eucaliptos estudados neste trabalho não teve diferença significativa nestes (Tabelas 2 e 3), não possibilitando, assim, a indicação do(s) tipo(s) de eucalipto mais favorável(eis) para o cultivo do shiitake. Em relação aos demais nutrientes, de acordo com Boyle (1998), a disponibilidade de simples carboidratos, micronutrientes ou vitaminas não limita a taxa de crescimento do fungo na madeira.

Os principais elementos inorgânicos das madeiras foram Ca, K e Mg. Expressando esses elementos em $\mathrm{mg} / \mathrm{kg}$ e convertendo o $\mathrm{K}_{2} \mathrm{O}$ para $\mathrm{K}$ através das massas moleculares (fator de conversão $=0,8298$ ), tem-se que o teor de Ca na madeira variou de $100-700 \mathrm{mg} / \mathrm{kg}$, o de K, de 330-664 mg/kg e o de Mg, de 100-200 mg/kg. O teor de $\mathrm{Na}$ variou de $136,67-393,33 \mathrm{mg} / \mathrm{kg}$ e o de $\mathrm{P}$ (convertendo $\mathrm{P}_{2} \mathrm{O}_{5}$ para $\mathrm{P}$ através do fator de conversão 0,4366) variou de $87,32-174,64 \mathrm{mg} / \mathrm{kg}$. A grande maioria dos valores obtidos de $\mathrm{Cu}$, Fe e Mn esteve abaixo de $50 \mathrm{mg} / \mathrm{kg}$ e de $\mathrm{Zn}$, abaixo de $100 \mathrm{mg} / \mathrm{kg}$. A magnitude dos valores encontrados nos diversos metais analisados, bem como a classificação dos elementos em principais, intermediários e traços, está de acordo com Fengel e Wegener (1984) nas diversas espécies de madeiras.

O uso de toras de eucalipto no cultivo de shiitake no Brasil é muito comum. No entanto, muitos produtores reclamam da sua produtividade inconstante e baixa. Queiroz (2002) relatou que a pequena quantidade de nutrientes na madeira associada à presença de microrganismos competidores possivelmente são os principais fatores associados às diferenças de desenvolvimento do shiitake no eucalipto. De acordo com Barros (1990), devido ao fato de o eucalipto ser espécie arbórea muito cultivada em solos pobres, ele permite acúmulo menor de nutrientes na madeira.

Montini (1997) verificou irregularidade na produção do shiitake em toras de eucalipto, em que algumas chegaram a produzir 660 g de cogumelo no primeiro fluxo, no entanto muitas nada produziram. De acordo com Shimoyama (1990), a composição química da madeira varia significativamente entre espécies, entre árvores de uma mesma espécie e, mesmo, entre partes de uma mesma árvore.

Revista Árvore, Viçosa-MG, v.35, n.2, p.183-192, 2011
Os macronutrientes fósforo, potássio e magnésio são necessários para o crescimento de diversos fungos (CHANG et al., 1997). Na Tabela 1, sabe-se que esses nutrientes estão presentes na madeira. Entre os microelementos mais estudados e essenciais para o crescimento de muitas espécies merecem destaque: ferro, zinco, manganês e cobre (MOLENA, 1986; MILES e CHANG, 1997).

Não há na literatura relatos que apontem a influência da concentração de sódio ou de manganês na produtividade de $L$. edodes. Os dados apresentados na Figura 1 (B e C) não foram suficientes para afirmar que esses nutrientes influenciaram na produtividade das linhagens de $L$. edodes. Prova disso são o E. saligna e o E. citriodora - embora estejam entre os eucaliptos que proporcionaram as melhores médias de produtividade do L. edodes no trabalho de Andrade (2007), suas médias de teores de sódio e de manganês diferiram entre si (Figura 1 BC). Possivelmente, as quantidades de nutrientes disponíveis nas madeiras foram suficientes para o cultivo de L. edodes. Com isso, o fato de um tipo de eucalipto ter maior disponibilidade desses nutrientes não lhe proporcionou vantagem na produção de basidiomas, conforme relatado por Andrade (2007).

Os principais elementos inorgânicos presentes na casca foram Ca $(13.900-29.000 \mathrm{mg} / \mathrm{kg})$; K, calculado a partir do $\mathrm{K}_{2} \mathrm{O}(1.494-2.323 \mathrm{mg} / \mathrm{kg})$; e $\mathrm{Mg}$ (3.500 - $9.300 \mathrm{mg} / \mathrm{kg})$. O teor de $\mathrm{Na}$ variou de $237-683 \mathrm{mg} / \mathrm{kg}$ e o de $\mathrm{P}$ calculado a partir do $\mathrm{P}_{2} \mathrm{O}_{5}$, de 43,7 - $349 \mathrm{mg} / \mathrm{kg}$; o Cu (<50 mg/kg), o Fe e o Zn ( $<100 \mathrm{mg} / \mathrm{kg})$ e o Mn $(<1.000 \mathrm{mg} / \mathrm{kg})$. As análises de amostras da casca revelam que esse material tem valores muito mais elevados de $\mathrm{Ca}, \mathrm{K}$ e $\mathrm{Mg}$ que os correspondentes encontrados na madeira (Tabela 1) e são concordantes com a conclusão de Allison et al. (2000). A casca tem muito mais componentes minerais que a madeira correspondente e o elemento predominante é o cálcio, conforme Fengel e Wegener (1984).

\section{CONCLUSÕES}

De acordo com os resultados, pode-se concluir que:

- O elemento S não foi detectado em nenhum dos tipos de eucalipto analisados, tanto na casca quanto na madeira, como esperado.

- Na madeira, o Mg, Na e Mn apresentaram diferenças de teor entre os tipos de eucaliptos avaliados. 
- Na casca, o K 2 O, C, Cu e Mn demonstraram possuir diferentes teores em função do tipo de eucalipto.

As cascas dos tipos de eucaliptos apresentaram teores de $\mathrm{Ca}, \mathrm{K}, \mathrm{Mg}$, Na, Mn e Fe superiores aos das correspondentes madeiras (o elemento predominante na casca foi o $\mathrm{Ca}$ ).

- Os elementos predominantes na madeira foram Ca, K, Mg e P.

\section{REFERÊNCIAS}

ALLISON, L. et al. Metal profiling of southeastern U.S. softwood and hardwood furnish. TAPPI Journal, v.83, n.8, p.97, 2000.

ANDRADE, M. C. N. et al. Mycelial growth of two Lentinula edodes strains in culture media prepared with sawdust extracts from seven eucalyptus species and three eucalyptus clones. Acta Scientiarum. Agronomy, v.30, n.3, p.333-337, 2008.

ANDRADE, M. C. N. Crescimento micelial, produção e características bromatológicas do shiitake em função de linhagens e de propriedades físicas e químicas de espécies e clones de eucalipto. 2007. 195f. Tese (Doutorado em Agronomia/Energia na Agricultura)-Faculdade de Ciências Agronômicas, Universidade Estadual Paulista, Botucatu, 2007.

ANDRADE, M. C. N. et al. Avaliação do crescimento micelial de linhagens de shiitake, da produção em toras de eucalipto e de alterações físicas da madeira. Acta Scientiarum Agronomy, v.29, n.1, p.23-27, 2007.

ANDRADE, M. C. N.; GRACIOLLI, L. A. Controle de fungos contaminantes no cultivo do cogumelo comestível shiitake em toros de eucalipto. Acta Scientiarum Agronomy, v.27, n.2, p.293-299, 2005.

ANDRADE, M. C. N. et al. Análise de um sistema de produção do cogumelo comestível shiitake na região de Ilha Solteira - SP. Cultura

Agronômica, v.11, n.1, p.109-123, 2002.

BARROS, N. F. Relação solo-planta. Viçosa, MG: Folha de Viçosa, 1990. 330p.
BOYLE, C. D. Nutritional factors limiting the growth of Lentinula edodes and other white-rot fungi in wood. Soil Biology \& Biochemistry, v.30, p.817-823, 1998.

BROWNING, B. L. The chemistry of wood. New York: John Wiley \& Sons, 1963. 689 p.

CHANG, S. T.; MILES, P. G. Edible mushrooms and their cultivation. Воса Raton: CRC Press, 1989. 345p.

EIRA, A. F.; MONTINI, R. M. C. Manual teórico prático de cultivo de cogumelo Shiitake (Lentinula edodes (Berk.) Pegler). Botucatu: Fundação de Estudos e Pesquisas Agrícolas e Florestais, 1997. 38p.

FENGEL, D.; WEGENER, G. Wood, chemistry, ultrastructure, reactions. New York: Walter de Gruyter, 1984. 613p.

LANARV. Laboratório de Referência Vegetal. Análise de fertilizantes e inoculantes: métodos oficiais. Brasília: Secretaria Nacional de Defesa Agropecuária, 1988. 104p.

MILES, P. G.; CHANG, S. T. Mushroom biology-concise basics and current developments. London: World Scientific, 1997. 177p.

MINHONI, M. T. A. et al. Cultivo de Lentinula edodes (Berk.) Pegler (Shiitake). 3.ed. Botucatu: FEPAF, 2007. 91p.

MOLENA, O. O moderno cultivo de cogumelos. São Paulo: Nobel, 1986.170p.

MOnTINi, R. M. C. Produtividade de shiitake (Lentinula edodes (Berk.) Pegler), no primeiro choque de indução, em função de características dos toros do Eucalyptus saligna, Sm, variáveis do ambiente e período de incubação. 1997. 61f. Dissertação (Mestrado em Agronomia/ Energia na Agricultura)-Faculdade de Ciências Agronômicas, Universidade Estadual Paulista, Botucatu, 1997.

PRZYBYLOWICZ, P.; DONOGHUE, J. Lentinula edodes grower's handbook: the art and science of mushroon cultivation. Dubuque: Kendall, 1990. 217p.

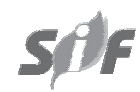

Revista Árvore, Viçosa-MG, v.35, n.2, p.183-192, 2011 
QUEIROZ, E. C. Efeito da suplementação mineral na conversão de energia e produtividade do Shiitake em toros de eucalipto. 2002. 73f. Dissertação (Mestrado em Agronomia/ Energia na Agricultura)-Faculdade de Ciências Agronômicas, Universidade Estadual Paulista, Botucatu, 2002.

SAN ANTONIO, J. P. Cultivation of the shiitake mushroom. HortScience, v.16, n.2, p.151-156, 1981.

SHIMOYAMA, V. R. Variações da densidade básica e características anatômicas e químicas da madeira em Eucalyptus sp. 1990. 93f. Dissertação (Mestrado em Ciência Florestal) - Escola Superior de Agricultura Luiz de Queiroz, Universidade de São Paulo, Piracicaba, 1990.

SILVA, J. C. Eucalipto: pesquisa amplia usos: perspectivas do setor florestal brasileiro.

Revista da Madeira, v.13, n.75, p.4-6, 2003.

SNEDECOR, G. W. E.; COCHRAN, W. G.

Statistical methods. 6.ed. Ames: Iwoa State University Press, 1972. 325p.
SOUZA, A. P. et al. Estudo da densidade básica da madeira de Eucalyptus microcorys. F. Muell, cultivado na região de Dionísio, MG. Revista Árvore, v.3., n.1, p.16-27, 1979.

TEIXEIRA, E. M. Caracterização isoenzimática e molecular de Lentinula edodes e avaliação da produção em função da espécie de eucalipto e clima. 2000. 123f. Tese (Doutorado em Biotecnologia/ Biotecnologia) - Instituto de Química, Universidade Estadual Paulista, Araraquara, 2000.

TOKIMOTO, K. Shiitake log cultivation. In: GUSH, R. (Ed.). Shiitake cultivation. Korea: MushWord, 2005. p.56-87.

TREVISAN, H. et al. Avaliação de propriedades físicas e mecânicas da madeira de cinco espécies florestais em função da deterioração em dois ambientes. Revista Árvore, v.31, n.1, p.93-101, 2007.

TSOUMIS, G. Science and technology of wood: structure, properties, utilization. New York: Van Nostrand Reinold, 1991. 494p. 